\title{
CONNECTED FILTERING BY RECONSTRUCTION: BASIS AND NEW ADVANCES
}

\author{
Michael H.F. Wilkinson \\ Institute of Mathematics and Computing Science, University of Groningen
}

\begin{abstract}
Openings-by-reconstruction are the oldest connected filters, and indeed, reconstruction methodology lies at the heart of many connected operators such as levelings. Starting out from the basic reconstruction principle of iterated geodesic dilations, extensions such as the use of reconstruction criteria, which constrain the reconstruction process, are discussed. The latter prevent linking distinct objects connected by narrow bridges during the reconstruction process, whilst maintaining as much edge preservation as possible. A far faster variant of filtering with reconstruction criteria is presented, which can be implemented in an $O(N)$ algorithm in stead of $O\left(N^{2}\right)$.
\end{abstract}

Index Terms - Mathematical morphology, filtering by reconstruction, reconstruction criteria.

\section{INTRODUCTION}

Connected filters [1] are strictly edge preserving morphological filters. All connected filters act by merging flat zones in images, and assigning new grey levels to them. Filtering by reconstruction is the oldest type of connected filter [2]. Reconstruction operators can be implemented by iterated geodesic dilations of some marker by the unit ball. In the case of openings-by-reconstruction, the marker is the result of an opening or erosion by some structuring element. After this, all image structures not removed entirely by the opening or erosion are reconstructed exactly, as shown in Fig. 1.

One problem of filters by reconstruction, and indeed all connected filters is the so-called leakage-problem. Leakage occurs when spurious thin bridges connect separate image regions, making them inseparable. This is clearly visible in Fig. 1(c), in which the fine striping pattern in the clothes is reconstructed, effectively connecting, e.g. the face with the shoulder. To counter this problem, a variant of reconstruction, using so-called reconstruction criteria, is described in [3]. This is shown in Fig. 1(d). The problem with this method is that the fast algorithms developed for ordinary reconstruction [4] no longer work.

In this paper the basics of reconstruction are first discussed, followed by a brief description of levelings, and the use of reconstruction criteria. After this a new formulation of reconstruction criteria is presented, leading to a much faster algorithm (up to 1,235 times on a 3 mega-pixel image). The two formulations are not identical in their results, but the new method is such a close approximation that the differences are negligible on all images tested.

\section{OPENINGS BY RECONSTRUCTION}

The basis of an opening by reconstruction is the reconstruction of image $f$ from an arbitrary marker $g$. This is usually defined using geodesic dilations $\bar{\delta}_{f}$ defined as

$$
\bar{\delta}_{f}^{1}(g)=f \wedge \delta(g) .
$$

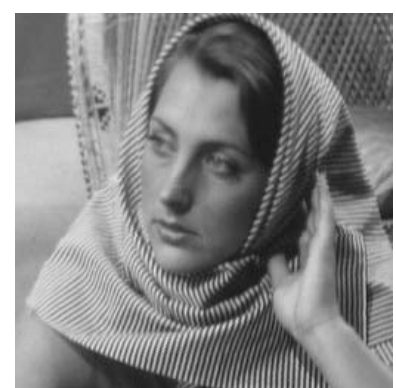

(a) original $f$

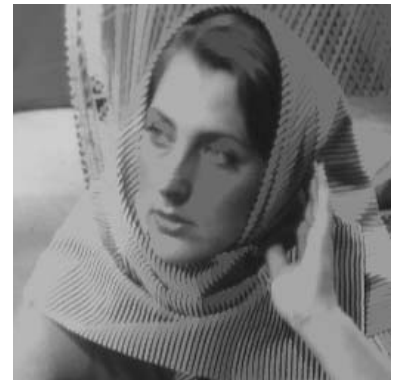

(c) reconstruction of $f$ by $g$

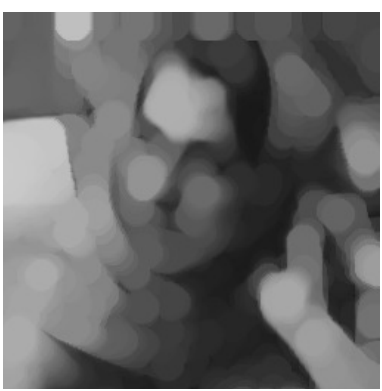

(b) marker $g=\gamma_{21} f$

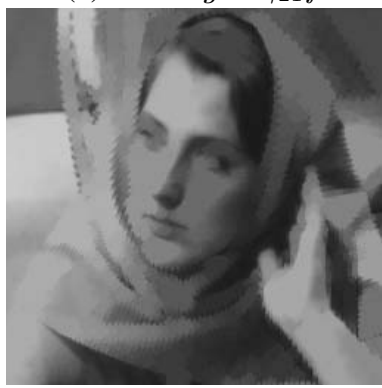

(d) reconstruction with criteria
Fig. 1. Grey scale image with marker computed by opening with Euclidean disc of diameter 21, and its reconstruction, and a reconstruction with reconstruction criteria.

This operator is used iteratively until stability, to perform the reconstruction $\rho$ i.e.

$$
\rho(f \mid g)=\lim _{n \rightarrow \infty} \bar{\delta}_{f}^{n} g=\underbrace{\bar{\delta}_{f}^{1} \ldots \bar{\delta}_{f}^{1} \bar{\delta}_{f}^{1}}_{\text {until stability }}(g) .
$$

In practice we apply $\bar{\delta}_{f}^{n}$ with $n$ the smallest integer such that

$$
\bar{\delta}_{f}^{n} g=\bar{\delta}_{f}^{n-1} g .
$$

What this process does in the binary case is reconstruct any connected component in $f$ which intersects some part of $g$. An opening by reconstruction is computed by selecting marker $g$ by

$$
g=\gamma_{X} f,
$$

in which $\gamma_{X}$ denotes an opening of $f$ by structuring element (S.E.) $X$. Reconstructing from this marker preserves any connected component in which $X$ fits at at least one position.

Computing a reconstruction from markers by direct implementation of (2) is simple enough, but very slow. It can readily be shown that its worst-case time complexity is $O\left(N^{2}\right)$, with $N$ the number 


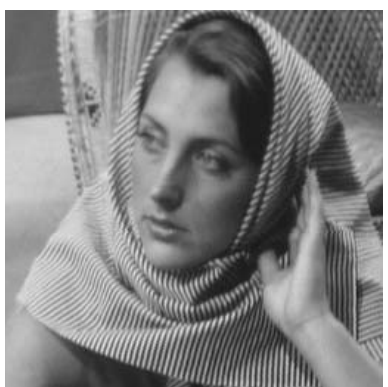

(a) original $f$

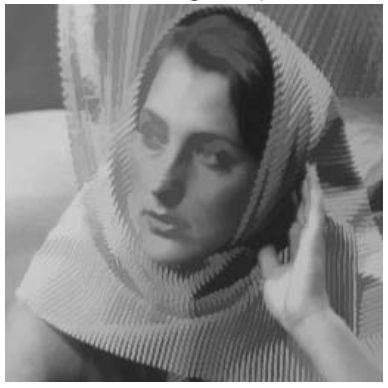

(c) leveling of $f$ by $g$

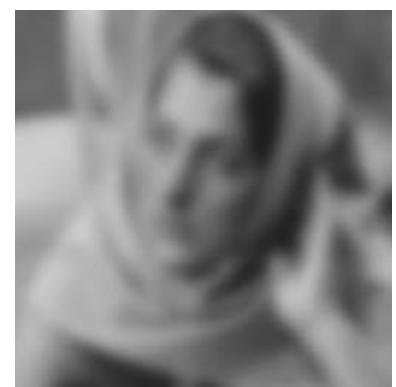

(b) marker $g=G_{16} * f$

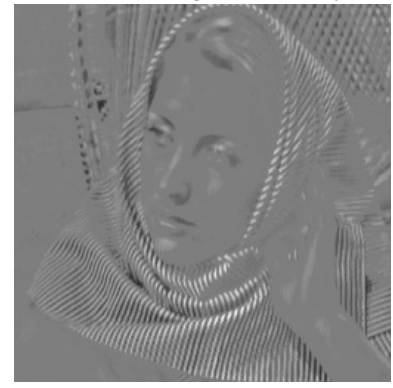

(d) texture image
Fig. 2. Levelings: Part (c) shows leveling of image (a) using marker obtained by Gaussian smoothing with $\sigma=16$ (b); (d) shows the texture signal obtained by subtracting (c) from (a).

of pixels. Low-order complexity (effectively linear) algorithms have been developed, notably by Vincent [4]. These are based on floodfilling, and should really be used in any implementation of reconstruction filters.

Closing-by-reconstruction can be defined by duality, i.e. a closing-by-reconstruction can be computed by inverting the image, performing the opening-by-reconstruction, and inverting the result.

\section{LEVELINGS}

Openings-by-reconstructions are anti-extensive, and closings-byreconstructions are extensive, removing bright or dark image details respectively. Meyer [5] proposed levelings as an auto-dual extension of reconstruction filters. In this case a marker is used which may lie partly above and partly below the image. In those regions where the image lies above the marker, the reconstruction proceeds as in the case of openings by reconstruction. Wherever the marker is above the original, reconstruction proceeds as in the case of closing-byreconstruction, i.e.

$$
(\lambda(f \mid g))(x)= \begin{cases}(\rho(f \mid g))(x) & \text { if } f(x) \geq g(x) \\ -(\rho(-f \mid-g))(x) & \text { if } f(x)<g(x)\end{cases}
$$

with $\lambda(f \mid g)$ the leveling of $f$ from $g$. Levelings allow edgepreserving simplification of images, by simultaneously removing bright and dark details, as seen in Fig. 2.

One application of levelings is in separating the coarse details in a "cartoon image" from the fine details in a texture channel [6]. Ideally, all fine detail should be in the texture channel (in Fig. 2(d)), but as can be seen in Fig. 2(c), leakage causes part of the fine texture to appear in the cartoon channel.

\section{ADDRESSING LEAKAGE}

A key problem in all connected filters is that distinct objects which are connected through spurious narrow bridges caused e.g. by noise cannot be separated readily. One approach is to use so-called contraction-based, second-generation connectivity [7]. Focusing on the binary case, we can rewrite reconstruction as

$$
\rho(X \mid M)=\bigcup_{x \in M} \Gamma_{x}(X)
$$

in which $\Gamma_{x}$ is the connectivity opening at $x$ which simply extracts the connected component of $X$ containing $x$, and yields $\emptyset$ if $x \notin X$, using regular (4 or 8) connectivity. Equation (6) states that the reconstruction of $X$ from $M$ is the union of all connected components of $X$ which have nonempty intersection with $M$. Second generation connectivity changes the way we extract connected components. To counter the leakage problem we use

$$
\Gamma_{B}^{\gamma}(X)= \begin{cases}\Gamma_{x}\left(\gamma_{B} X\right) & \text { if } x \in \gamma_{B} X \\ \{x\} & \text { if } x \in X \backslash \gamma_{B} X \\ \emptyset & \text { otherwise }\end{cases}
$$

with $\gamma_{B}$ a structural opening by a ball $B$. We can simply replace $\Gamma_{x}$ in (6) by $\Gamma_{x}^{\gamma}$ to use the adapted connectivity. This however boils down to doing a reconstruction of an opening of the image by $B$ [8]. The diameter of $B$ determines the thickness of any bridge which is considered wide enough for the reconstruction process to proceed.

An alternative is the use of reconstruction criteria [3]. Reconstruction from markers using reconstruction criteria are based on a simple adaptation of (2). In this scheme it is possible to introduce a reconstruction criterion, which prevents the flooding process to "leak" through narrow bridges linking objects together. This is done by performing an opening by a ball $\gamma$ between each pair of conditional dilations, i.e.

$$
\rho_{\gamma}(f \mid g)=\lim _{n \rightarrow \infty} \bar{\delta}_{f}^{n} g=\underbrace{\bar{\delta}_{f}^{1} \gamma_{B} \ldots \bar{\delta}_{f}^{1} \gamma_{B} \bar{\delta}_{f}^{1} \gamma_{B}}_{\text {until stability }}(g) .
$$

This means that at every step of the iteration, the growing region is restricted to a union of balls of the diameter of $B$. Denoted more compactly we have

$$
\rho_{\gamma}(f \mid g)=\left(\bar{\delta}_{f} \gamma_{B}\right)^{n} g .
$$

In contrast to standard reconstruction, no fast algorithm for reconstruction with reconstruction criteria have been proposed. This is a serious drawback, as the iterative solution has complexity $O\left(N^{2}\right)$. This is a problem, because the reduced leakage can yield better cartoon/texture separation, as shown in Fig. 3. Practically, all fine lines are restricted to the texture channel, unlike in Fig. 2(c) and (d).

Note that this method is not strictly speaking a connected filter anymore, even though it is closely related. Because it is not a connected filter, it cannot be expressed in terms of a family of connectivity openings. This can readily be shown, because connected regions of the image which are reconstructed from different markers may partially overlap, which is not possible in the connected case. An example can be seen in Fig. 4

\subsection{An approximation}

As stated above, the reconstruction using reconstruction criteria restricts the growing region to a union of translated versions of ball $B$, 


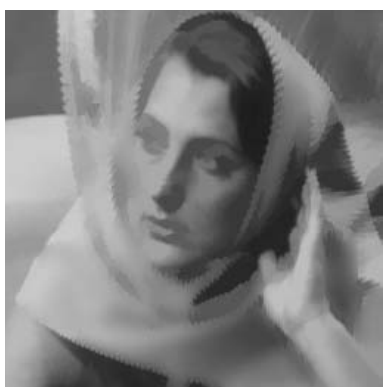

(a) cartoon

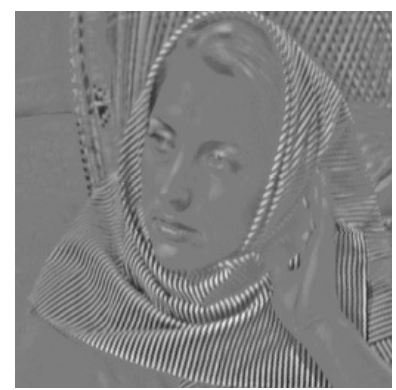

(b) texture
Fig. 3. Cartoon/texture decomposition using leveling with reconstruction criteria, with original and marker image as in Fig. 2(a) and (b), and diameter of $B$ set to 3 pixels.

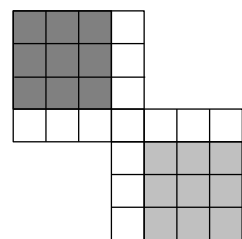

(a)

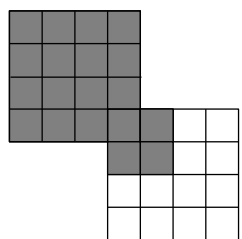

(b)

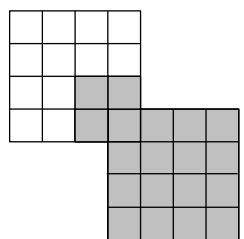

(c)
Fig. 4. Reconstruction using reconstruction criteria may yield overlapping results, when starting from different markers: (a) Region $f$ consisting of two intersecting squares, showing two markers in different shades of grey; (b) Result of reconstruction with criteria using $3 \times 3$ square both for $\gamma_{B}$ and $\bar{\delta}_{f}^{1}$ using upper marker. It can easily be verified that after reaching the dark grey area, the iteration ceases. Part (c) same using lower marker.

by the definition of structural openings. One might say the reconstruction process now "floods" the area to be reconstructed with a viscous liquid consisting of balls defined by the structuring element $B$, similar to the ideas in [9]. We can study the centroids of these balls by rewriting (9) as

$$
\rho_{\gamma}(f \mid g)=\left(\bar{\delta}_{f} \delta_{B} \epsilon_{B}\right)^{n} g .
$$

At each step, the centroids of these balls are obtained immediately before the dilation by $B$, i.e. just after the erosion by $B$. Because the erosion is applied to a subset of $f$ at each step, we can easily see that these can only lie in the region defined by the erosion $\epsilon_{B} f$.

Therefore, let us consider an alternative reconstruction method, which reconstructs the appropriate parts of $\epsilon_{B} f$ without reconstruction criteria, and then corrects this result by performing the last step of (10) except for the erosion, to correct for the initial erosion of $f$. The idea is to effectively change the order of processing, to obtain an ordinary reconstruction, sandwiched between a few simple structural operators, instead of the costly iterations in (8) through (10). This change of processing order does not guarantee that the result will be the same, but it should be very similar.

Thus, we first erode both the marker $g$ and the image $f$ by $B$. We then reconstruct the erosion of $f$ using the erosion of $g$ as marker. It can easily be verified that the first step in (10) is an erosion of $g$ as well. Therefore, any parts of a marker removed in this step are also removed by the original algorithm. The process reconstructs any

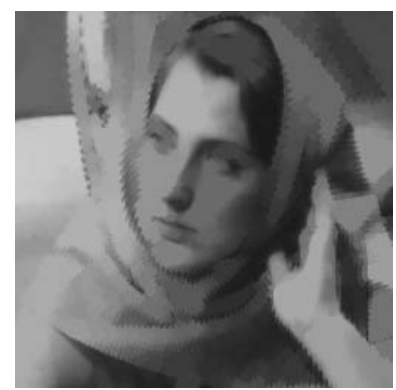

(a) $\rho_{\gamma}(f \mid g)$

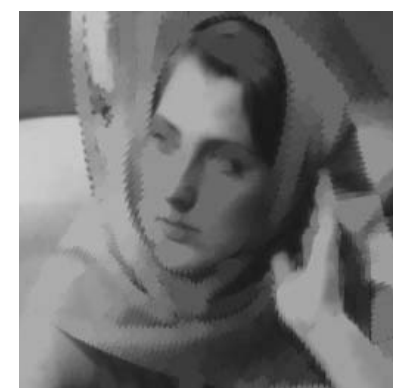

(b) $\rho_{\gamma}^{\prime}(f \mid g)$

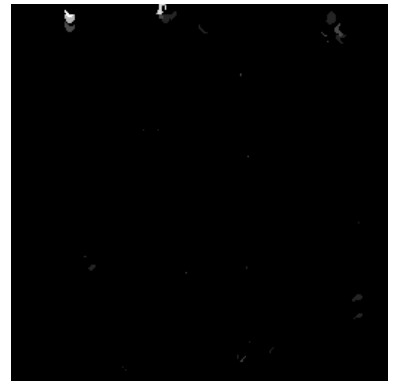

(c) $\rho_{\gamma}(f \mid g)-\rho_{\gamma}^{\prime}(f \mid g)$

Fig. 5. Detail of two variants of reconstruction with reconstruction criteria (a) and (b), with original and marker image as in Fig. 1(a) and (b), and diameter of $B$ set to 7 pixels. The difference image (c) has the contrast stretched by a factor of 32 .

connected component of $\epsilon_{B} f$ intersected by $\epsilon_{B} g$. We now have the collection of centroids reached by the flooding process. To obtain the set of balls needed, rather than just the centroids, we now dilate the reconstructed region, followed by a last conditional dilation. We can therefore define this approximate operator $\rho_{\gamma}^{\prime}$ as

$$
\rho_{\gamma}^{\prime}(f \mid g)=\bar{\delta}_{f} \delta_{B} \rho\left(\epsilon_{B} f \mid \epsilon_{B} g\right)
$$

The cost of this operator is that of two erosions, one dilation, one conditional dilation (all $O(N)$ ) and an ordinary reconstruction (also $O(N)$ in practice).

This approach is only an approximation of reconstruction criteria, and we find minor differences between the two methods, as shown in Fig. 5. On a number of outdoor scenes, fewer than $1 \%$ of pixels showed any difference, and generally by less than 6 grey levels. In a few cases some larger grey-level differences were found along the image edges, possibly because the implementation of the two algorithms treat image borders differently. A more detailed analysis is needed to determine the precise differences between the two approaches.

\section{EXPERIMENT}

Both algorithms were implemented using the erosion and dilation algorithm for arbitrary S.E. from [10]. The reconstruction algorithm used in $\rho_{\gamma}^{\prime}$ was based on the Max-Tree algorithm [11], which has worst-case complexity $O(G N)$ with $G$ the number of grey levels, but which is effectively linear for most natural images. Timings were performed on a Core 2 Quad machine running at $2.4 \mathrm{GHz}$ with $2 \mathrm{~GB}$ of RAM. The program used only one core. A series of openings by Euclidean disks with diameters 11, 21, 41, 81, and 161 pixels, were computed as markers for the reconstruction. One street scene of 3 


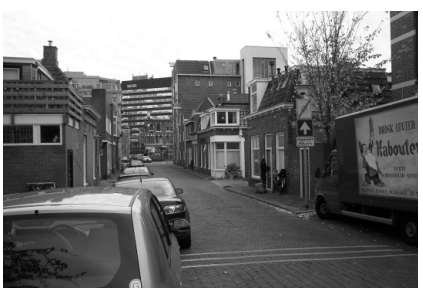

street

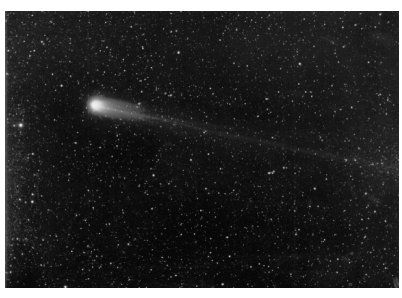

comet
Fig. 6. The test images.

mega-pixel and one astronomical image (a comet) of the same size were chosen as test images for the timings (see Fig. 6). Timings were performed as a function of the diameter of the diameter of the (Euclidean) ball $B$ used for the reconstruction criterion. Timings are shown in Fig. 7.

As expected, the new approach was far faster, outperforming the old method by two to three orders of magnitude, except when the same S.E. was used for both marker generation and criterion. In the latter case the method reduces to an ordinary opening followed by a geodesic dilation. Computing times for the old method went up to almost 11 minutes, whereas the slowest run on the new method was about $1 \mathrm{~s}$. The largest speed ratio was 1,235 times. When used on a fixed marker image, the computing time of the new algorithm rises approximately linearly with the diameter of $B$, due to the complexity of dilation and erosions from [10]. If square S.E. are used, this increase can be avoided by appropriate decomposition. Fluctuations in the iterative approach depend both on S.E. size as above, but also on the varying number of iterations needed before convergence. This is strongly influenced by image content, as can be seen by the differences in computing time between the "street" (509 s) and "comet" images ( $238 \mathrm{~s})$ at ball diameter of 11 . The new method does not have such a strong dependence on image content.

\section{CONCLUSION}

Reconstruction operators, including levelings are powerful operators with many applications in image processing. The leakage problem can be overcome by using reconstruction criteria or contractionbased connectivity. The method from [3] suffers from the fact that no fast implementation is available. In this paper a new variant of reconstruction criteria is proposed, which is practically identical to the approach of [3], but is orders of magnitude faster. In the future this method may be used in any application requiring cartoon/texture decomposition of images.

\section{REFERENCES}

[1] P. Salembier and J. Serra, "Flat zones filtering, connected operators, and filters by reconstruction," IEEE Trans. Image Proc., vol. 4, pp. 1153-1160, 1995.

[2] J. C. Klein, Conception et réalisation d'une unité logique pour l'analyse quantitative d'images, Ph.D. thesis, Nancy University, France, 1976.

[3] I. R. Terol-Villalobos and D. Vargas-Vázquez, "Openings and closings with reconstruction criteria: a study of a class of lower and upper levelings," J. Electron. Imaging, vol. 14, no. 1, pp. 013006, 2005.
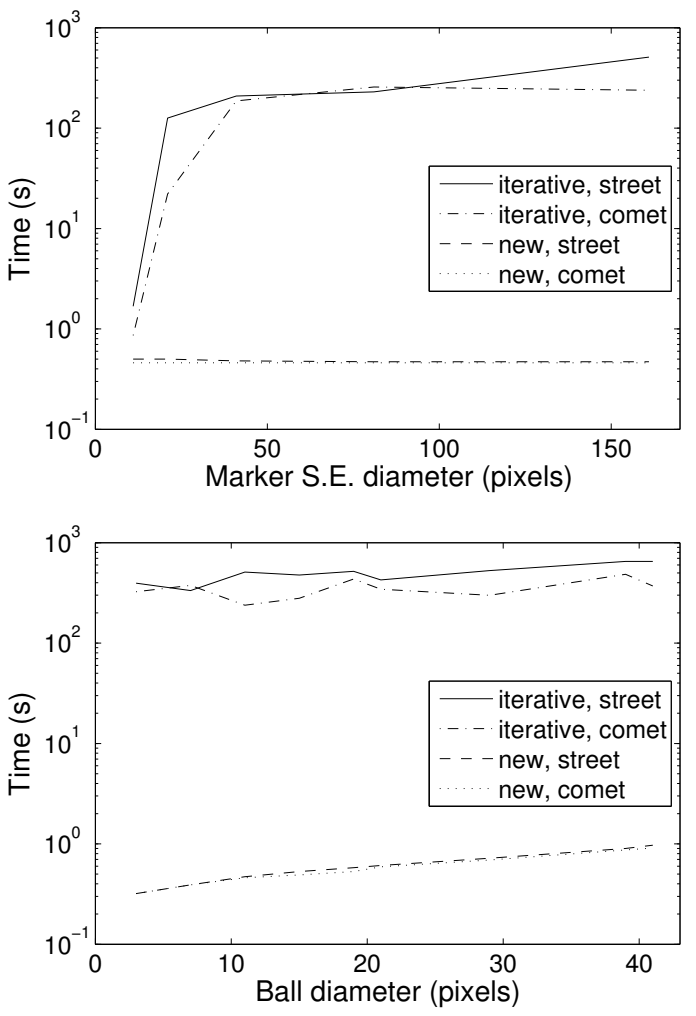

Fig. 7. Timings of iterative algorithm for $\rho_{\gamma}(f \mid g)$ versus the new method for $\rho_{\gamma}^{\prime}(f \mid g)$ : The top panel shows the computing times for a criterion using a ball of diameter 11 as a function of the S.E. diameter used to obtain the marker. The bottom panel shows the computing times for a fixed marker (obtained with and S.E. of 161 pixels diameter) as a function of the diameter of the ball $B$ used for the criterion.

[4] L. Vincent, "Morphological grayscale reconstruction in image analysis: application and efficient algorithm," IEEE Trans. Image Proc., vol. 2, pp. 176-201, 1993.

[5] F. Meyer, "Levelings, image simplification filters for segmentation," J. Math. Imag. Vis., vol. 20, no. 1-2, pp. 59-72, 2004.

[6] P. Maragos and G. Evangelopoulos, "Leveling cartoons, texture energy markers, and image decomposition," in Proc. Int. Symp. Math. Morphology (ISMM) 2007, 2007, pp. 125-138.

[7] U. Braga-Neto and J. Goutsias, "A theoretical tour of connectivity in image processing and analysis," J. Math. Imag. Vis., vol. 19, pp. 5-31, 2003.

[8] M. H. F. Wilkinson, "Attribute-space connectivity and connected filters," Image Vis. Comput., vol. 25, pp. 426-435, 2007.

[9] J. Serra, "Viscous lattices," J. Math. Imag. Vis., vol. 22, no. 2-3, pp. 269-282, 2005.

[10] E. R. Urbach and M. H. F. Wilkinson, "Efficient 2-D grayscale morphological transformations with arbitrary flat structuring elements.," IEEE Trans. Image Proc., vol. 17, pp. 1-8, 2008.

[11] P. Salembier, A. Oliveras, and L. Garrido, "Anti-extensive connected operators for image and sequence processing," IEEE Trans. Image Proc., vol. 7, pp. 555-570, 1998. 